\title{
Herausforderung und Chance für die Psychiatrie
}

\author{
Stefan Kesslera, Pascal Scholla, Christian Bernath ${ }^{b}$ \\ ${ }^{a}$ Continget Beteiligung \& Führung AG; ${ }^{b}$ Facharzt für Psychiatrie und Psychotherapie, Mitglied FMH
}

An einer psychischen Erkrankung leiden und erfolgreich im Arbeitsleben stehen oft kein Widerspruch. Wenn Lösungen gelingen, bleiben sie jedoch meist im Verborgenen. Ein Plädoyer an Psychiaterinnen und Psychiater, Kontakt zur Arbeitswelt zu suchen, neue Konzepte zu erproben und bei der Bekämpfung der beruflichen Stigmatisierung psychisch Erkrankter vor der eigenen Haustür nicht Halt zu machen.

\section{Die Ausgangslage: betrübliche Zahlen und ein wenig bekannter Erfolgsausweis}

In der Fachwelt, Politik und Wirtschaft ist bekannt, dass psychische Leiden zu grossen menschlichen, sozialen und wirtschaftlichen Problemen führen - in der Gesellschaft allgemein und ganz konkret bei den Betroffenen und ihren Angehörigen. Ein eindrücklicher Hinweis hierzu ist die Entwicklung der Invalidenrenten aufgrund psychischer Erkrankungen: Betrug deren Zahl im Jahr 1986 noch rund 24000, beläuft sie sich 30 Jahre später auf gut $122000 .{ }^{1}$ Für die Schweiz schätzte die OECD die jährlichen Kosten auf etwa 3,2 Prozent der Wirtschaftsleistung oder 19 Milliarden Franken.

Weniger verbreitet ist das Wissen um eine andere statistische Grösse: $75 \%$ bis $80 \%$ aller Personen im erwerbsfähigen Alter, die an einer mittelschweren oder schweren psychischen Erkrankung leiden, stehen im Arbeitsprozess. ${ }^{2}$ Diese Zahl ist, auch im Vergleich mit anderen Ländern, erstaunlich hoch und grundsätzlich erfreulich; im Zusammenhang mit oben genannter Renten-Entwicklung wirkt sie überraschend, ist aber kein Widerspruch.

\section{«Bei uns ist wenig möglich»}

Viele Mitarbeiterinnen und Mitarbeiter erleben während des beruflichen Lebens eine psychische Erkrankung. In Unternehmen hören wir oft, dass eine solche, auch nach erfolgreicher Behandlung, ein Karrierehemmer bleibt, nach dem Motto: «Wer schon bei der aktuellen Belastung an die Leistungsgrenze gelangt, wird sicher nicht befördert.» Dies unabhängig davon,

\section{Un défi et une chance pour la psychiatrie}

Nombre de personnes souffrent de troubles psychiques pendant leur vie professionnelle. Dans le quotidien professionnel prévaut l'idée selon laquelle ce type de maladie revient à perdre sa capacité de rendement pour toute la vie; pourtant, les statistiques révèlent aussi un autre aspect: beaucoup parviennent malgré tout à rester engagés dans le processus de travail. Le cercle vicieux du jeu de cache-cache professionnel est une explication conceptuelle de cette apparente contradiction.

Pour les psychiatres, la situation de départ dans les entreprises est importante. Elle permet de concevoir une approche de chaque cas individuel. L'idée principale est la suivante: dans beaucoup de situations, ne serait-il pas avantageux que le médecin traitant s'investisse plus dans le système employeur-employé? Plusieurs arguments suggèrent que cela irait dans l'intérêt du patient. Cette démarche génère aussi des opportunités pour la psychiatrie, que ce soit au niveau technique ou économique. Les troubles psychiques ne sont pas seulement source d'importants problèmes humains, ils entraînent aussi des difficultés économiques, pour les personnes concernées, les entreprises et les assurances. Qui est à même de relever ce défi, sinon la psychiatrie?

Pour conclure, les auteurs dressent le tableau d'une pratique de cette spécialité qui «construirait des passerelles professionnelles». Ils posent la question: cette démarche est-elle une utopie ou une ligne de conduite réaliste, qu'il convient d'adopter à travers des étapes plus ou moins ambitieuses?

ob zwischen Erkrankung und Berufskontext ein $\mathrm{Zu}$ sammenhang besteht. Zudem sind zwar viele Führungskräfte der Auffassung, dass für Menschen mit psychischen Beeinträchtigungen etwas getan werden sollte. Gerade ihre Abteilung eigne sich allerdings nicht dafür, weil eine uneingeschränkte Leistungsfähigkeit notwendig sei: «Bei uns ist leider wenig möglich!» Ein weiteres Phänomen: Vielen Personalfachleuten ist zwar bekannt, dass der frühzeitige Einbezug des 
Arbeitsumfeldes die Bewältigung einer psychischen Erkrankung positiv beeinflussen könnte. Paradoxerweise wird die Früherkennung aber gerade im Arbeitsumfeld kaum wahrgenommen.

Wie ist es mit dieser Ausgangslage erklärbar, dass so viele psychisch Erkrankte arbeiten, während im beruflichen Alltag der Eindruck überwiegt, eine solche Erkrankung sei erstens "ein Problem der andern» und zweitens - wenn sie denn eintritt - quasi gleichbedeutend mit einem lebenslangen Verlust an Leistungsfähigkeit?

\section{Eine konzeptionelle Erklärung - der Teufelskreis des beruflichen Versteck- spiels}

Der Teufelskreis des beruflichen Versteckspiels (Abb. 1) bietet eine modellhafte Erklärung:

In den Unternehmen läuft ein Prozess ab, der dazu führt, dass Mitarbeitende aller Stufen psychische Problematiken am Arbeitsplatz nicht bzw. so spät wie nur möglich offenlegen. Obwohl dieses Verhalten sowohl für das Unternehmen als auch für die Mitarbeitenden gesamthaft nachteilig ist, besteht für die einzelnen Teile des Systems - Mitarbeitende, Vorgesetzte, Zuständige für Personalselektion, strategische Gremien - kein wirksamer Anreiz, den Teufelskreis zu durchbrechen.

Die Kernaussagen des Modells:

Viele Mitarbeiterinnen und Mitarbeiter erleben während des beruflichen Lebens eine psychische Erkrankung.

- Weil Mitarbeitende ihre Symptome und Beeinträchtigungen verstecken, wird frühes, präventives Handeln - z.B. eine Anpassung der Arbeitsumgebung, des Pensums, der Aufgaben oder auch der Zusammenarbeit - nicht möglich.

- Weil Mitarbeitende mit positivem Heilungsverlauf oder erfolgreichem Umgang mit der Krankheit d.h. mit kurzer oder gar nie eintretender Arbeitsunfähigkeit - sich am Arbeitsplatz nicht zu erkennen geben, werden die Häufigkeit psychischer Erkrankungen sowie die Zahl gelungener beruflicher Lösungen unterschätzt.

- Weil von jenen Fällen, in denen lange Absenzen und schwierige Arbeitskonstellationen auftreten, fast jeder im Unternehmen erfährt - «hast Du gehört, X hat ein Burn-out!»-, wird die Schwere der Fälle systematisch überschätzt.

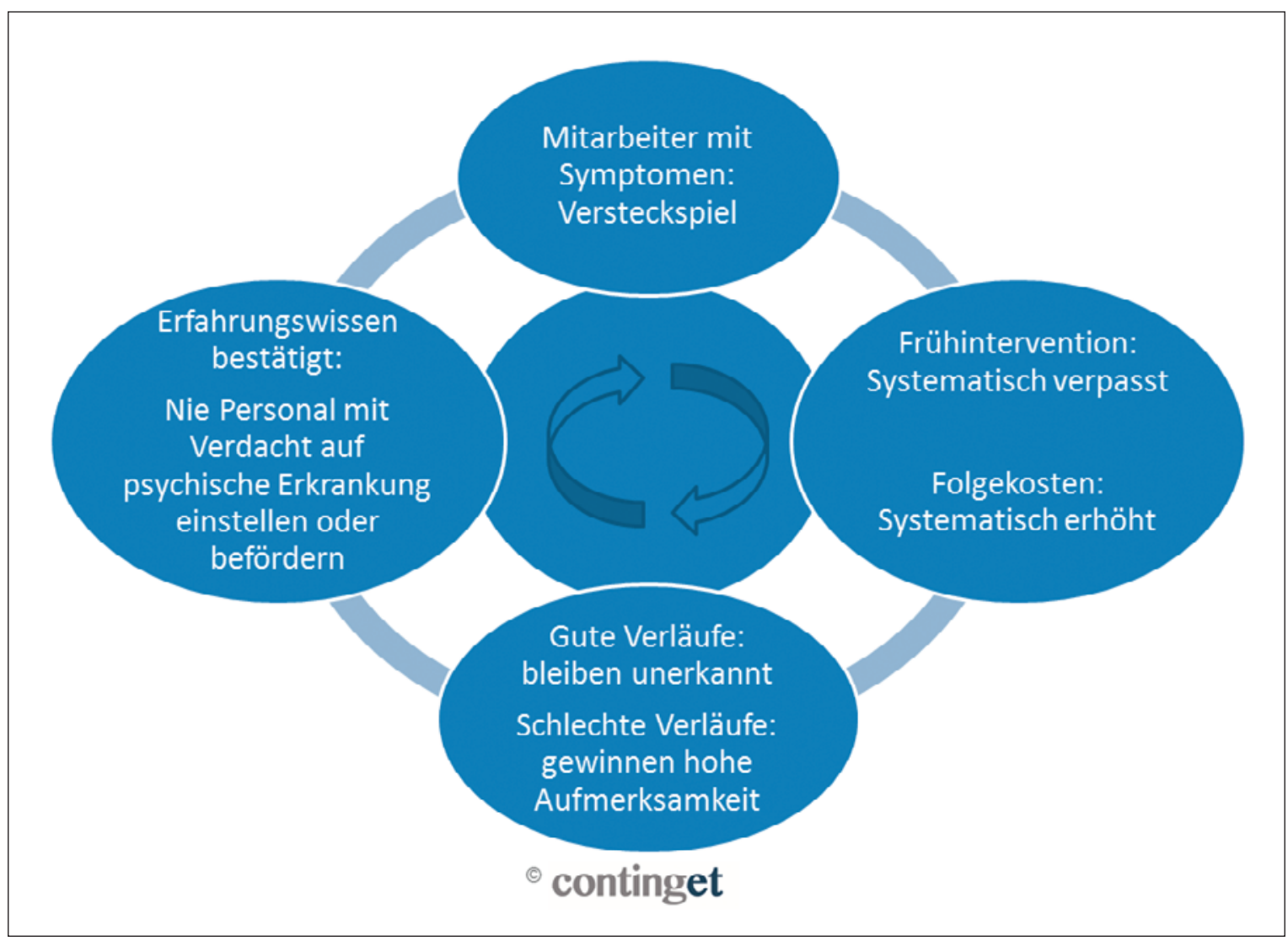

Abbildung 1: Der Teufelskreis des beruflichen Versteckspiels psychischer Erkrankungen in Unternehmen. 
- Weil das negative Erfahrungswissen von Vorgesetzten und Entscheidungsgremien dadurch immer wieder bestätigt wird, werden Menschen mit bekannter oder vermuteter psychischer Erkrankung nicht eingestellt und nicht befördert, auch wenn sie längst wieder gesund sind.

- Weil das so ist, verstecken Mitarbeitende ihre Symptome.

Alle Beteiligten verhalten sich nach bestem Wissen und Gewissen. Dies verleiht dem Teufelskreis Stabilität. Die Tatsache, dass ein beträchtlicher Teil der Folgekosten frühestens mittelfristig auftritt und in anderen Systemen - z.B. in der IV oder Pensionskasse -, fördert die Problemwahrnehmung nicht.

\section{Herausforderungen und Chancen für die Psychiatrie}

In der Arbeitswelt treffen wir also auf eine vertrackte Ausgangslage, die sich durch nachvollziehbare und schwierig zu durchbrechende Beweggründe aller Beteiligten im Einzelfall auszeichnet. Inwiefern ist diese Situation nun für Psychiaterinnen und Psychiater relevant? Im Folgenden beantworten wir diese Frage, im Sinne einer ersten Anregung, aus den zwei Blickwinkeln «Einzelfall» und «Branche».

\section{Ansatzpunkte für Psychiaterinnen und Psychiater als «Behandelnde Instanz» im Einzelfall}

Unsere Kernanregung in einem Satz: Wäre es nicht in zahlreichen Einzelfällen von Vorteil, wenn sich die behandelnde Psychiaterin, der behandelnde Psychiater oder auch der allenfalls involvierte Hausarzt aktiver in das Arbeitgeber-Arbeitnehmer-System des Patienten einbringt?

\section{Weil Mitarbeitende ihre Symptome und} Beeinträchtigungen verstecken, wird frühes, präventives Handeln nicht möglich.

Folgende Argumente sprechen dafür, dass ein solches Vorgehen den Patientennutzen massgeblich erhöhen kann:

- Es wird frühzeitig und sorgfältig geklärt, ob die Arbeitssituation als Ressource im Behandlungskonzept genutzt werden kann - oder ob sie eher als Genesungsrestriktion zu betrachten ist. Die Behandlung wird entsprechend optimiert.

- Das Arbeitgebersystem erhält notwendige Informationen (nur diese, und nur mit Zustimmung des
Patienten!): z.B. betreffend krankheitsspezifischen Heilungsverläufen (Zeitraum, Auswirkungen auf den Arbeitsalltag). So werden berufliche Teil-Potentiale besser nutzbar und Restriktionen, z.B. ein drohender Stellenverlust, sichtbarer - was es ermöglicht, mit ihnen umzugehen.

- Vorbehalte und Ängste beim Arbeitgeber können abgebaut bzw. versachlicht werden. Auch für die Patienten in ihrem Bestreben, beruflich langfristig eine Perspektive zu haben, ist dies ein grosser Vorteil.

- Der Patient erfährt eine umfassende, lebensweltorientierte Behandlung, in der auch berufliche Faktoren professionell beurteilt, berücksichtigt und bearbeitet werden - im Wissen darum, dass beruflicher Erfolg ein wichtiges Element der Genesung sein kann.

Ein aktiver, von ärztlicher Seite angeregter Kontakt mit dem Arbeitgeber stellt hohe Ansprüche an die Wahrnehmung der eigenen Rolle und Aufgabe. Der Arzt ist grundsätzlich verpflichtet, die Schweigepflicht

\section{Vorbehalte und Ängste beim Arbeitgeber} können abgebaut bzw. versachlicht werden.

einzuhalten. Dies ist immer noch die Grundlage für eine offene und vertrauensvolle Beziehung zwischen Patient und Arzt. Massgeschneiderte Lösungen - z.B. Teilvollmachten zur Gewährleistung des Daten- und Patientenschutzes - sind aber möglich, insbesondere, wenn auf Stufe der gesamten Branche Instrumente und Wissen entwickelt und bereitgestellt werden.

\section{Ansatzpunkte für die Psychiatrie als Branche und Arbeitgeberin}

Aus Sicht der Autoren bieten sich aufgrund der komplexen Situation im Arbeitsleben - Stichwort «Teufelskreis» - für die Psychiatrie als Branche fachliche und ökonomische Chancen:

Fachlich ist einerseits die Erhöhung des Patientennutzens, wie oben beschrieben, ein zentrales Ziel. Gleichzeitig bietet der beschriebene Ansatz die Möglichkeit, die psychiatrische Tätigkeit - in Fortsetzung der sozialpsychiatrischen Tradition - um inhaltlich attraktive Elemente zu erweitern: eine grössere Lebensweltorientierung, die Konzeption wirklich umfassender Behandlungskonzepte sowie die Erschliessung und Anwendung von zusätzlichem Wissen, z.B. in spezifischen Berufsfeldern, bieten gerade auch jungen Psychiaterinnen und Psychiatern spannende Perspektiven. Ökonomisch bietet eine vermehrte Arbeitswelt-Orientierung Potential. Betriebe, Privat- und Sozialversiche- 
rungen sowie die Volkswirtschaft leiden an den steigenden Kosten beruflicher Ausgliederung psychisch erkrankter Menschen - wer, wenn nicht die Psychiatrie, hat das Know-how, um diesem Problem zu begegnen? Gelingt es der Branche, sich als Akteurin zur Verbesserung der Gesundheits- und Erwerbssituation $\mathrm{zu}$ positionieren, wird dies auch zu einer höheren Branchenprosperität führen. In den Tarifsystemen sind die Entgelte für den Einbezug des Patientenumfeldes weiter zu verbessern. Dies ergänzend zu den heutigen Regelungen, die in vielen Fällen bereits entsprechende Interventionen ermöglichen. Selbstbewusstes Aushandeln der Rahmenbedingungen, sei dies im Einzelfall oder auf Stufe der Tarife, ist angezeigt und angemessen.

\section{Die psychiatrische Praxis als «Berufs- Brückenbauerin" - eine Utopie?}

Stellen wir uns die Gruppenpraxis «P+ AG» vor. In ihr arbeiten 5 Psychiaterinnen und Psychiater, die sich bei Patienten, bei grossen Arbeitgebern der Region und bei der kantonalen IV-Stelle den Ruf erarbeitet haben, zur Genesung und beruflichen Integration ihrer Patienten beizutragen. Der regelmässige Kontakt zum Arbeitgeber gehört zum Standard-Behandlungspfad. Das familiäre Umfeld ist einbezogen. Sie bieten innovative, berufsbezogene Leistungen an -z.B. einen runden Tisch zur Standortbestimmung und eingliederungsorientierte Gutachten - und verfügen über spezifisches Know-how. Ein Partner hat sich in Eingliederungsrecht weitergebildet und kennt die Finanzierungsmöglichkeiten bei der IV à fonds, ein anderer hat ein CAS in Case Management absolviert.

Die Praxis «P+ AG» verfügt für sich selbst über innovative interne Prozesse und Leitlinien. So wird bei allen wichtigen internen betrieblichen Fragen geprüft, welche Auswirkungen die Entscheide auf die physische und psychische Gesundheit der eigenen Mitarbeitenden haben. Versicherungen wurden so ausgewählt, dass alle Mitarbeitenden von einem kleinen, aber feinen Gesundheitsmanagement profitieren. Der Umgang mit eigenen erkrankten Mitarbeitenden ist professio-

\section{In den Tarifsystemen sind die Entgelte} für den Einbezug des Patienten-Umfeldes weiter zu verbessern.

nell: So wurde die heikle Frage, ob und wie sich die allfällige Erkrankung eines Psychiaters auf seine Patientenarbeit auswirkt, diskutiert und der Umgang damit klar geregelt. Auf ihrer Homepage weist «P+ AG» darauf hin, dass sie sich als Arbeitgeberin aktiv gegen das Stigma «einmal psychisch krank = lebenslang leistungsgemindert» einsetzt.

\section{Mehr beruflicher Erfolg als Ziel}

Ist eine solche Psychiatriepraxis, als "Berufs-Brückenbauerin", eine realistische fachliche und unternehmerische Perspektive - oder eine Utopie? Aus Sicht der Autoren hat die beschriebene Marschrichtung, in grösseren oder auch kleineren Schritten, ein doppeltes Potential: Einerseits, mehr psychisch erkrankten Menschen in mehr Unternehmungen mehr langfristigen beruflichen Erfolg zu ermöglichen. Andererseits, Psychiatern und Hausärzten erweiterte berufliche Entwicklungsperspektiven zu eröffnen.

Bildnachweis

(c) Continget 2015 